\title{
Systematic Preoperative Complementary Tests in Elective Minor and Medium Pediatric Surgery: Is it Time to Revalue?
}

\author{
B Gafsi ${ }^{1}$, S Toumi ${ }^{1}$, A Zouaoui ${ }^{2}$, K Moula ${ }^{1}$, A Ksia ${ }^{2, *}$, M Gahbiche ${ }^{1}$ \\ ${ }^{1}$ Department of Anesthesia, University Hospital Fattouma Bourguiba, Monastir, Tunisia \\ ${ }^{2}$ Department of Pediatric Surgery, University Hospital Fattouma Bourguiba, Monastir, Tunisia
}

\section{Abstract}

Introduction: Prescription of routine complementary tests preoperatively has become a tradition for all practitioners. These tests are ordered frequently but their contribution doesn't seem that significant. The aim of our study is to find out whether these systematic preoperative tests, prescribed for children undergoing elective minor and medium surgeries, were truly indicated or unjustified.

Patients and Methods: We conducted a prospective, observational study including 230 children admitted for scheduled surgery with minor to medium bleeding risk. Several parameters were collected: the surgery and anesthesia characteristics, complementary preoperative tests, the tests prescriber, their usefulness and the cost of the prescription.

Results: The mean age was 3 years old. The majority (95\%) belonged to ASA I class. Twenty-eight percent of these children couldn't walk yet. Eighty-two percent of the undergone surgeries had a minor bleeding risk. In $74 \%$ of cases, the chosen technique was a general anesthesia combined to local anesthesia. For the 230 patients studied, 857 complementary tests were requested. A systematic blood assessment was performed for all the patients. Only $35,86 \%$ of the realized tests were actually indicated. Excluding blood group, analysis of other tests showed that only $28,71 \%$ of them were abnormal. The total cost of the complementary tests was 8919.1 dinars of which $64,14 \%$ were spent on non-indicated tests.

Conclusion: The systematic routine prescribing of blood tests must be forsaken to switch to a selective and rational prescription. Recommendations and guidelines may afford a precious help to organize the blood tests prescription procedure.

\section{Introduction}

Pre-operative evaluation is a fundamental for all patients proposed for a surgery. It reduces per-operative risks (surgical or anesthetic) $[1,2]$.

This evaluation is based firstly on a good clinical investigation and the physical examination, then on the complementary tests.

The aims of these tests are essentially to obtain information about patients' aptitude for anesthesia and surgery, to detect unknown pathologies that may increase the operative risks and whose diagnosis may modify the pre and per-operative management [3].

Various recommendations that tend to rationalize and limit this type of prescription are published in many countries. Because of the low incidence of associated cardiovascular and pulmonary diseases, the pediatric problem is dominated by the worry of detecting a disorder of hemostasis. This kind of disorder may cause a bleeding complication associated to the anesthetic or the surgical technique.

The aim of our study is to evaluate the prescription of preoperative complementary tests in the operatory unit of the pediatric surgery and to determine the cost of the useless prescribed tests.

This is an observational prospective single-center study, conducted during four months (January-April 2018) in the operating unit of the pediatric surgery department in Fattouma Bourguiba Hospital, Monastir. It is a first phase evaluation of professional practices. During this phase no training was done about the prescription of preoperative tests (neither for the surgeons nor the anesthetist doctors). org/10.15344/2455-2364/2020/162
The inclusion criteria were: age less than 14 years old, American Society of Anesthesiologists (ASA) I or II class, scheduled surgery, minor to medium bleeding risk surgery, surgery practiced with general anesthesia combined or not to loco-regional anesthesia (LRA), pre evaluation in the consultation of anesthesia made by an anesthetist doctor (resident or senior).

The exclusion criteria were: age higher than 14 years old, ASA III class or more, absence of prior pre-anesthetic evaluation, surgical emergency, surgery at high risk of bleeding, surgery underwent exclusively with LRA, unavailability of an anesthetist doctor for data collection.

Once the child was admitted at the pediatric surgery operating room (after validation of the inclusion criteria), several parameters were collected by the anesthetist doctor using a pre established record card. This card included two parts:

\section{A first part concerning}

Information provided by the parents: the demographic data (age, gender, socioeconomic level and the type of the social insurance if

"Corresponding Author: Prof. Amine Ksia, Department of Pediatric Surgery, University Hospital Fattouma Bourguiba, Monastir 5000, Tunisia; E-mail: amineks@yahoo.fr

Citation: Gafsi B, Toumi S, Zouaoui A, Moula K, Ksia A, et al. (2020) Systematic Preoperative Complementary Tests in Elective Minor and Medium Pediatric Surgery: Is it Time to Revalue? Int J Pediatr Neonat Care 6: 162. doi: https://doi.

Copyright: (c) 2020 Gafsi et al. This is an open-access article distributed under the terms of the Creative Commons Attribution License, which permits unrestricted use, distribution, and reproduction in any medium, provided the original author and source are credited. 
Citation: Gafsi B, Toumi S, Zouaoui A, Moula K, Ksia A, et al. (2020) Systematic Preoperative Complementary Tests in Elective Minor and Medium Pediatric Surgery: Is it Time to Revalue? Int J Pediatr Neonat Care 6: 162. doi: https://doi.org/10.15344/2455-2364/2020/162

Page 2 of 5

present), the pathological history as well as the ASA class and the ability of walking.

\section{A second part containing data related to}

The surgery: its type, whether it is ambulatory, its risks essentially the bleeding risk according to literature and to the local practitioners.

The anesthesia: the anesthetic technique, the type of the realized LRA and its localization: central (intrathecal morphine, caudal block) or peripheral block (ilioinguinal block, paraumbilical block, pudendal block, penile block).

Complementary preoperative blood tests: the tests, the prescriber: anesthetist doctor or surgeon, the usefulness of the prescription (whether the requested test is indicated or not).

The usefulness of the tests was deduced from: the type of the surgery, the patient's history (presence of a known disease, acquisition of walking skills) and the recommendations of the SFAR 2012 which are based on the GRADE approach (grading of recommendations assessment, development and evaluation) to attest their relevance on general population (adult and pediatric).

Other collected data: anomalies in the tests results, if changes were done in the pre-operative management when there were anomalies, the total economic cost of the prescription, the economic participation of the parents.

The results of the study were recorded and analyzed using the Statistical Package for the Social Sciences SPSS STATISTICS 20 software. A descriptive analysis was conducted on the entire population. The qualitative variables are expressed in percentages. Quantitative variables are expressed as mean +/- standard deviation. Comparative analyzes of qualitative variables were performed by a Chi2 test or Fisher's exact test if the number was less than 5 . The threshold of significance retained was $\mathrm{p}<0.05$.

\section{Results}

During the period of the study, 310 patients were studied: 230 were included and 80 were excluded. The average age of the children was 3 years (ranging from 3 months to 14 years old). Twenty-eight percent of these children had not walked yet. The male/female ratio was: 3, 69 . The majority of the children included belonged to ASA I class (95\% of cases). A "low" socioeconomic status was noted in more than half of the included population (51.3\%).

The different surgical procedures were: thoracic, abdominal, urological, proctologic, hernia repair procedures, and $66,08 \%$ of these surgeries were ambulatory. The majority of the realized surgeries $(82 \%)$ had a minor bleeding risk.

In $74 \%$ of cases, the chosen technique was a general anesthesia combined to LRA. An exclusive general anesthesia was practiced in the rest of cases. The realized LRA was a peripheral block in $79 \%$ of cases and a central block in the rest of cases.

For the 230 patients included in our study, 857 complementary tests were made. The prescription rate of different tests is represented by figure 1. A systematic blood assessment: Blood Grouping (BG,) Complete Blood Count (CBC), coagulation: Prothrombin Time (PT and Activated Partial Thromboplastin Time (APTT) were performed for all the patients.

In the majority of cases $(95,22 \%)$ the prescription of the complementary tests was made by the surgeon and $4,78 \%$ by anesthetist. Most of the complementary tests $(87.8 \%)$ were conducted in the public sector.

\section{Analytical study}

Only $35,86 \%$ of the realized tests had an indication. The ordering of the systematic blood tests (BG, CBC, PT and APTT) had no

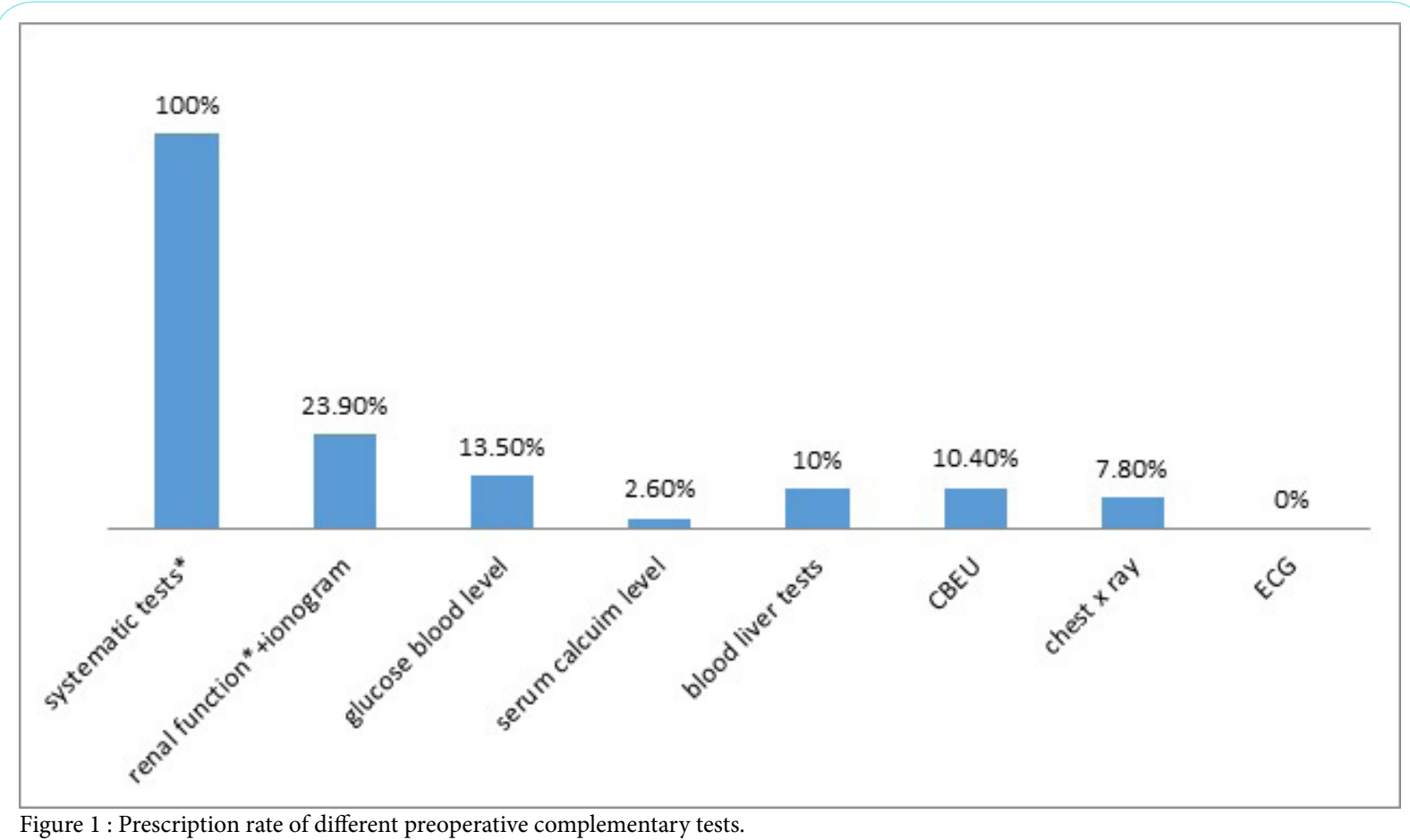

Figure 1 : Prescription rate of different preoperative complementary tests. 
Citation: Gafsi B, Toumi S, Zouaoui A, Moula K, Ksia A, et al. (2020) Systematic Preoperative Complementary Tests in Elective Minor and Medium Pediatric Surgery: Is it Time to Revalue? Int J Pediatr Neonat Care 6: 162. doi: https://doi.org/10.15344/2455-2364/2020/162

Page 3 of 5

indication in most cases with a statistically significant difference. The case was not always similar for the other blood tests (Table 1).

\begin{tabular}{|l|l|l|l|}
\hline & Non indicated & Indicated & $\mathrm{P}$ \\
\hline BG & $81,3 \%$ & $18,7 \%$ & $<0,001$ \\
\hline CBC & $58 \%$ & $42 \%$ & $<0,001$ \\
\hline PT & $99,6 \%$ & $0,4 \%$ & $<0,001$ \\
\hline APTT & $71,6 \%$ & $28,4 \%$ & $<0,001$ \\
\hline Blood gGlucose blood & $90,37 \%$ & 9,63 & $<0,001$ \\
\hline Renal fonction & $71,12 \%$ & $28,88 \%$ & $<0,001$ \\
\hline Serum calcuim & $100 \%$ & $0 \%$ & $<0,001$ \\
\hline Liver blood tests & $4 \%$ & $96 \%$ & $<0,001$ \\
\hline CBEU & $0 \%$ & $100 \%$ & $<0,001$ \\
\hline Chest x ray & $66,66 \%$ & $33,34 \%$ & $<0,001$ \\
\hline
\end{tabular}

Table 1: Complementary tests ordering' rate.

For non-indicated tests: the prescription was made by the surgeon for all cases with a statistically significant difference $(p<0.001)$.

The low rate of the prescription made by the anesthetist doctor had an indication in all cases with a statistically significant difference ( $\mathrm{p}$ $<0.001)$.

For the ASA I class, the non-indicated tests are more frequent than the indicated ones with a statistically significant difference $(p<0.001)$.

Excluding blood group, analysis of other tests (627) showed that only $28,71 \%$ of them are abnormal (Table 2 ).

The abnormalities noted in non-indicated tests did not require any changes in the pre-operative management for the majority of cases with a statistically significant difference $(p<0.001)$.

The abnormalities that changed the preoperative management were more frequent for the indicated tests than for non-indicated tests with a statistically significant difference $(\mathrm{p}<0.001)$.

The total cost of the complementary tests was 8919.1 dinars of which 5720.7 dinars $(64,14 \%)$ were spent on non-indicated tests. The participation of the parents in the total cost is 4872,22 of which $2817,36(57,82 \%)$ are spent on non-indicated tests.

\section{Discussion}

The prescription of complementary tests has been considered for a longtime as a systematic step integrated into the pre-operative evaluation. This systematization stopped implicitly the reflexion step before any prescription. Therefore, the tests are prescribed without considering their indication, their usefulness and their cost.

The prescription of complementary tests has been considered for a longtime as a systematic step integrated into the pre-operative evaluation. This systematization stopped implicitly the reflexion step before any prescription. Therefore, the tests are prescribed without considering their indication, their usefulness and their cost.

Our studies corroborate these facts. We found out indeed that more than the half $(64,14 \%)$ of the prescribed tests had no indication. Even when the tests results (indicated or not indicated) were pathological, a change in the pre-operative management was infrequent: 45 cases $(25 \%)$.

Thus systematic complementary tests prescription prolonged the procedure of pre-operative preparation and increased the expenses: almost 9000 dinars in our study.

This amount of money is alarming especially since our study was conducted in a developing country facing an economic crisis. As a result, an urgent and radical change in this routine prescription attitude is to be done.

This change must certainly come from the foundation of a scientific basis to guide the prescription. Hence the concept of establishing local recommendations to will streamline the ordering of preoperative complementary tests.

Our study will be the substratum of these recommendations since it took into consideration the particularities of the children undergoing various surgeries and reflected the real conditions in the pediatric surgery department of Fattouma Bourguiba Hospital. In fact the first obstacle encountered when starting our study was the lack of studies in the literature concerning the prescription of preoperative tests specifically in pediatric population [4-7].

Either for the children or for the adults, the complementary tests are an essential part of the preoperative evaluation (if they are indicated). They must be specific and targeted at the clinical signs found by the interrogation and the physical examination. This was approved by Garcia et al. who considered that these tests have the purpose of confirming and documenting the conditions likely to affect the course of the surgery and of the anesthesia that it requires [1]. These tests must remain secondary at the clinical evaluation so they must not be systematic [8-12].

Actually this was not the case in our study since all our patients had a minimum of four complementary tests: BG, PT, APTT and CBC.

\begin{tabular}{|l|l|l|l|l|}
\hline & Non indicated & Indicated & Total & P \\
\hline ASA Class & & & & \\
\hline ASA I & $550 / 820(67,08 \%)$ & $270 / 820(32,92 \%)$ & $820 / 857(95,69 \%)$ & $<0,001$ \\
\hline ASA II & $0 / 37$ & $37 / 37$ & $37 / 857(4,31 \%)$ & $<0,001$ \\
\hline Normal test & $262 / 447(58,62 \%)$ & $185 / 447(41,38 \%)$ & $447 / 627(71,29 \%)$ & $<0,005$ \\
\hline Abnormal test & $64 / 180(35,6 \%)$ & $116 / 180(64,4 \%)$ & $180 / 627(28,71 \%)$ & $<0,001$ \\
\hline Abnormal test without specific preoperative management & $58 / 64(90,62 \%)$ & $77 / 116(66,37 \%)$ & $135 / 180(75 \%)$ & $<0,001$ \\
\hline Abnormal test with specific preoperative management & $6 / 64(9,38 \%)$ & $39 / 116(33,63 \%)$ & $45 / 180(25 \%)$ & $<0,001$ \\
\hline
\end{tabular}

Table 2: Relation between the existence of an indication and the ASA class, normal or abnormal test. 
Citation: Gafsi B, Toumi S, Zouaoui A, Moula K, Ksia A, et al. (2020) Systematic Preoperative Complementary Tests in Elective Minor and Medium Pediatric Surgery: Is it Time to Revalue? Int J Pediatr Neonat Care 6: 162. doi: https://doi.org/10.15344/2455-2364/2020/162

Page 4 of 5

\section{Prevalence of non-indicated tests}

Most of the prescribed tests $(64,14 \%)$ in our study did not have any indication. This rate is obviously huge and had an impact on the economic and scientific levels. This fact highlighted the absence of our approach's conformity with the international recommendations $[13,14]$.

Several other studies found a high prevalence of non-indicated complementary tests. For example, in a study conducted in Pennsylvania (United States) involving 3111 patients proposed for an ambulatory surgery, it was shown that $52.9 \%$ of all these patients had at least one non-indicated biological test performed pre-operatively [15].

For ASA class interference with indications, our study showed that more than the half $(67,08 \%, 550$ out of 820$)$ of the complementary tests requested for patients belonging to ASA I class had no indication with statistically significant difference $(\mathrm{p}<0.001)$. For patients belonging to ASA II class, the indicated tests are more frequent than the nonindicated tests with a statistically significant difference $(p<0.001)$ These facts pointed out that the ASA class was obviously correlated with the indication of the different complementary tests [16-18].

Comparable results were found in a Brazilian study concerning 1063 patients proposed for elective surgery. In fact, $41.9 \%$ of the realized complementary tests for patients belonging to ASA I class had no indication while only $17,72 \%$ of the realized complementary tests for patients belonging to ASA II class had no indication [1].

\section{Prevalence of abnormal results}

The abnormalities encountered in the indicated tests were more frequent than those discovered incidentally in the non-indicated tests with a statistically significant difference $(\mathrm{p}<0.001)$.

Even when complementary tests were indicated (in 301 cases) the abnormality rate was 116 (38.53\% of all indicated tests and $18.5 \%$ of all complementary tests). Besides in 77 cases $(66.37 \%$ of the abnormal test results) no particular management was required.

In this context Gampel et al. studied a cohort that included 46,977 patients proposed for scheduled inguinal hernia repair and found that $61.6 \%$ of patients had at least one abnormal result: hematological in $39.3 \%$ of cases and biochemical in $40.1 \%$ of cases. Despite of these abnormalities, repair of the hernia was performed in $100 \%$ of cases [19].

\section{The Problem of Cost}

Our study found a total complementary tests cost of almost 9000 dinars for only 230 patients. The cost of the unnecessary tests was: 5720.7 dinars. Thus, useless prescriptions caused not only a delay of the pre-operative procedure but also an excessive economic waste. Vogt and Henson conducted a retrospective study made of medical records showing that estimated savings of approximately 80,000 dollars annually could be obtained simply by eliminating nonindicated pre-operative tests for 5100 studied patients [20].

\section{The concept of Recommendations}

These recommendations concerning pre-operative complementary tests do not follow international standards. Each country and each institution can state its own local recommendations depending on its population and its working method. But they all share the same principle stating that the systematic prescription of the complementary tests must be abandoned and replaced by a selective and rational prescription, based on the history of the patient, the clinical examination and the type of the surgery [8].

In addition to the SFAR, several anesthesia' societies around the world are trying to establish a clear approach in the indication of the different preoperative tests [21-23].

Therefore, a development of clear recommendations is necessary for an effective way to manage the preoperative tests prescription. A local intra-hospital network must convey these recommendations once they are established, which will facilitate the sharing information and reduce unnecessary prescription. As in Ontario for example, hospitals had adopted the Ontario Preoperative Grill, recommended by the Ontario Preoperative Testing Group Advisory Committee of Recommendations [24].

But the establishment of the recommendations requires a very large number of studies and reviews. So our study by a sample of 230 patients presented only an initiation to a "revolution" against the systematic prescription of complementary tests.

\section{Limitations of Study}

First, a low sampling was studied (230 patients only). But as mentioned, our study will play the role of an initiation. Second, we were unable to make a refined statistical evaluation of the hemostasis tests due to the non-consideration of platelet count as an independent test: it is only practiced as a part of the $\mathrm{CBC}$ in both public and private laboratories. Then, the evaluation of the prescribers seemed to have a selection bias since the high rate of non-indicated prescriptions made by the surgeon is explained by his frequent solicitation.

\section{Conclusion}

In summary, our study demonstrated the overuse of pre-operative tests. Our findings along with previous research, suggested that unnecessary complementary tests performed during the pre-operative preparation of patients is still frequent, reflecting the uncertainty of indications and the lack of guidelines. Therefore, we concluded that these tests needed to be ordered not as a routine procedure, but in a scientific and rational way based on clinical history, physical examination and surgical procedures risk.

\section{Competing Interests}

The authors declare that they have no competing interests.

\section{References}

1. Garcia AP, Pastorio KA, Nunes RL, Locks GF, Simões de Almeida MC, et al. (2014) Indication of preoperative tests according to clinical criteria: need for supervision. Rev Bras Anestesiol 64: 54-61.

2. Van Klei WA, Moons KG, Rutten CL (2002) The effect of outpatient preoperative evaluation of hospital in patients on cancellation of surgery and length of hospital stay. Anesth Analg 94: 644-649.

3. Siriussawakul A, Nimmannit A, Rattana-arpa S, Chatrattanakulchai S, Saengtawan $P$, et al. Evaluating Compliance with Institutional Preoperative Testing Guidelines for Minimal-Risk Patients Undergoing Elective Surgery. Biomed Res Int 2013: 835426. 
Citation: Gafsi B, Toumi S, Zouaoui A, Moula K, Ksia A, et al. (2020) Systematic Preoperative Complementary Tests in Elective Minor and Medium Pediatric Surgery: Is it Time to Revalue? Int J Pediatr Neonat Care 6: 162. doi: https://doi.org/10.15344/2455-2364/2020/162

Page 5 of 5

4. Marwell JG, Heflin MT, McDonald SR (2018) Preoperative Screening. Clin Geriatr Med 34: 95-105.

5. Johansson T, Fritsch G, Flamm M, Hansbauer B, Bachofner N, et al. (2013) Effectiveness of non-cardiac preoperative testing in noncardiac elective surgery: a systematic review. Br J Anaesth 110: 926-939.

6. Mauck KF, Sundsted KK (2015) Update in perioperative medicine: evidence published in 2014. Ann Intern Med 162: 111-116.

7. Kazimierczak S, Kazimierczak A, Rynio P, Żukowski M (2015) Preoperative Evaluation; New Attempt. Pol Przegl Chir 87: 644-654.

8. Serafini G, Ingelmo PM, Astuto M, Baoncini S, Borrometi F, et al. (2014) Preoperative evaluation in infants and children : Recommendations of the Italian Society of Pediatric and Neonatal Anesthesia and Intensive Care (SARNePI). Minerva Anestesiol 80: 461-469.

9. Munro J, Booth A, Nicholl J (1997) Routine preoperative testing: a systematic review of the evidence. Health Technol Assess 1: 1-62.

10. Pasternak RL, Arens JF, Caplan RA (2002) Practice advisory for preanesthesia evaluation: a report by the American Society of Anesthesiologists Task Force on Preanesthesia Evaluation. Anesthesiology 96: 485-496.

11. Apfelbaum JL, Connis RT, Nickinovich DG, Pasternak LR, Arens JF, et al. (2012) Practice advisory for preanesthesia evaluation: an updated report by the American Society of Anesthesiologists Task Force on Preanesthesia Evaluation. Anesthesiology 116: 522-538.

12. Kumar A, Srivastava $U$ (2011) Role of routine laboratory investigations inpreoperative evaluation. J Anaesthesiol Clin Pharmacol 27: 174-179.

13. Yazıcı H, Daskaya H, Dogan S, Haberal I, Ciftci T, et al. (2014) Patient specific orroutine preoperative workup in septoplasty: which one is costeffective? Eur Arch Otorhinolaryngol 271: 305-309.

14. America Society of Anesthesiologists Task Force on Preanesthesia Evaluation (2002) Practice advisory for preanesthesia evaluation: a report by the American Society of Anesthesiologists Task Force on Preanesthesia Evaluation. Anesthesiology 96: 485-496.

15. Onuoha OC, Hatch MB, Miano TA, Fleisher LA (2015) The incidence of unindicated preoperative testing in a tertiary academic ambulatory center: a retrospective cohort study. Perioper Med 4: 14

16. Lira RP, Nascimento MA, Kara-José M, Arieta CE (2003) Predictive value of preoperative exams in facectomies. Rev Saude Publica 37: 197-202.

17. Correll DJ, Bader AM, Hull MW, Hsu C, Tsen LC, et al. (2006) Value of preoperative clinic visits inidentifying issues with potential impact on operating room efficiency. Anesthesiology 105: 1254-1259.

18. Halaszynski TM, Juda R, Silverman DG. Optimizing postoperative outcomes with efficient preoperative assessment and management. Crit Care Med 32: 576-586.

19. Benarroch-Gampel J, Sheffield KM, Duncan CB, Brown KM, Han Y, et al. (2012) Preoperative Laboratory Testing in Patients Undergoing Elective, Low-Risk Ambulatory Surgery. Ann Surg 256: 518-528.

20. Vogt AW, Henson LC (1997) Unindicated Preoperative Testing: ASA Physical Status and Financial Implications. Clin Anesth 9: 437-441.

21. Ferrando A, Ivaldi C, Buttiglieri A, Pagano E, Bonetto C, et al. (2005) Guidelines for preoperative assessment: impact on clinical practice and costs. Int J Qual in Health Care 17: 323-339.

22. O'Neill F, Carter E, Pink N, Smith I (2016) Routine preoperative tests for elective surgery: summary of updated NICE guidance. BMJ 354: 3292.

23. Arnold MJ, Beer J (2016) Preoperative evaluation: A time-saving algorithm J Fam Pract 65: 702-710.

24. Bryson GL, Wyand A, Bragg PR (2006) Preoperative testing is inconsisten with published guidelines and rarely changes management. Can J Anaesth 53: 236-241. 